\title{
Phase field study on crystal orientation effects in eutectoid phase transformation
}

\author{
Dong-qiao Zhang, Ya-jun Yin, * Jian-xin Zhou, and Zhi-xin Tu \\ State Key Laboratory of Materials Processing and Die \& Mould Technology, Huazhong University of Science and Technology, Wuhan 430074, \\ China,
}

\begin{abstract}
In this present work, a multi-phase field model was used to simulate the eutectoid transformation process, and on the basis of the nucleation model that was previously proposed by our research team, anisotropic and orientation relationship models were introduced to study the growth mechanism of the pearlite lamellae with anisotropy. It was found that the growth direction of the pearlite lamellae is related to its orientation and spacing. In the process of lamellar growth, deflection growth of pearlite will appear along with the adjustment of lamellar spacing, and the deflection angle is equal to the orientation difference between the austenite and the pearlite. Comparison between experimental and numerical results indicates a good consistency in pearlite morphology.
\end{abstract}

Key words: simulation; phase field; lamellar growth; pearlite morphology; growth direction
CLC numbers: TP391.99
Document code: A
Article ID: 1672-6421(2017)05-435-08

\begin{abstract}
$\mathrm{A}$ t present, most of the literature focused only on the isotropic eutectoid growth process, with less research on the eutectoid nucleation process and anisotropy. In the previous study, Zhang, et al. ${ }^{[1]}$ proposed a cooperative nucleation model, but did not consider anisotropy for the eutectoid growth. However, in studies by Zhi ${ }^{[2]}$ and Miyamoto ${ }^{[3]}$, it was found that eutectic and eutectoid growth process does include anisotropy.

As early as the $1950 \mathrm{~s}$, Smith ${ }^{[4]}$ studied the orientation of ferrite nuclei at the austenite grain boundary, and it was observed that the ferrite nuclei have a certain relationship with the orientation of austenite grains. Guo et al ${ }^{[5]}$ studied the kinetics and crystallography of the complex sedimentary surface nucleation of the intergranular pearlite in $\mathrm{Fe}-\mathrm{Mn}-\mathrm{C}$, and found that the incoherent $\mathrm{MnS}$ in the austenite cannot be strong nucleation position of pearlite, unless the transition time is extended. There is no special orientation relationship between lamellar ferrite and austenite matrix, but there
\end{abstract}

\footnotetext{
*Jian-xin Zhou

Male, born in 1975, Ph.D. and Professor. His research interests mainly focus on the computer applications in foundry industry, especially on casting process simulation and intelligent manufacturing for foundry enterprises.

E-mail: zhoujianxin@ hust.edu.cn
}

Received: 2017-08-15; Accepted: 2017-09-26 is a Pitsch-Petch orientation relationship between the ferrite and cementite of pearlite. Furuhara et al. ${ }^{[6]}$ added a small amount of $\mathrm{S}, \mathrm{V}$ and $\mathrm{N}$ to Fe-2Mn- $(0.13,0.2) \mathrm{C}$, and analyzed the effect of intergranular inclusions or precipitates on the kinetics of ferrite transformation at temperatures between $973-823 \mathrm{~K}$ and the orientation relationship between the inclusion and austenite, ferrite was obtained by similar experiments ${ }^{[7]}$. The phase concentration, dislocation density of austenite and ferrite/bainite/ martensite, lattice distortion and interstitial carbon atoms in austenite were investigated by Wixess et al. ${ }^{[8]}$ using X-ray diffraction techniques. The lattice distortion and the gap between the carbon atoms will affect the anisotropy of the phase. The phenomenon of ferrite nucleation on non-metallic inclusions was observed by Cheng et al. ${ }^{[9]}$. When the ferrite is at an elevated temperature, it presents an isolated island. The discoid ferrite is derived from the nucleation of the ferrite on the inclusions. It associated with the larger degree of subcooling, and with the parent austenite into a fixed orientation of growth. In 2010 , Cheng et al. ${ }^{[10]}$ observed the nucleation phenomenon of ferrite in the surface, edge and corner of the austenite grain boundary, and analyzed the mechanism of ferrite nucleation under three-dimensional morphology. When ferrite nucleated on the boundary surface of austenite grains, most of the ferrite nuclei were flat ellipsoid, some of which are plate-like or pyramidlike. When they nucleated on the edge of the grains, the 
morphology presented triangular pyramid or triangular double cone. When the nucleation position is at the corner of the grain boundary, it shows irregular shape. In 2013, the nucleation of ferrite and the three-dimensional kinetics of ferrite from austenite in low-carbon micro-alloyed high-strength steels were studied systematically by high temperature confocal laser microscopy, hot-pressing and electron backscatter diffraction experiments. Then Cheng et al. ${ }^{[1]}$ used continuous interface and computer aided technology to reconstruct the three-dimensional morphology of ferrite, and found that the anisotropy of ferrite can be obtained from its 3D morphology. Wang et al. ${ }^{[12]}$ studied the interfacial structure with Bagaryatsky orientation of ferrite/ cementite by approximating the repositioned lattice simulation. When the ferrite/cementite crystal orientation presents a specific relationship, the structural steps and mismatched dislocations can be formed on the interface, but this step is not an intrinsic structure and has no specific size, so the two-phase interface can be fluctuated within a certain range. This causes the ferrite/ cementite to have no macroscopic habitat. This phenomenon is conducive to bending growth of pearlite lamellar structure.

All of the above studies, both in terms of nucleation mechanism and orientation relationship, relate to the anisotropy of ferrite and cementite. Considering the anisotropy of the lamellar phase can lead to better understanding of the orientation relationship between austenite, ferrite, cementite and pearlite. In this work, with T8 steel as the research material, based on the nucleation model of proposed by our research team ${ }^{[1]}$ and the introduction of anisotropy and orientation relationship, the growth evolution process of pearlite lamellae was simulated. Then the nucleation sites of pearlite lamellae were discussed.

\section{Model and parameter conditions}

\subsection{Phase field model}

In this work, the multi-phase field model was used to simulate the eutectoid transformation process. The total free energy function is shown in equation (1). Equations (2) and (3) are interface energy density and chemical energy density, respectively.

$$
\begin{gathered}
F\left(\left\{\phi_{\alpha}\right\},\left\{\vec{c}_{\alpha}\right\}\right)=\int_{\Omega}\left\{f^{\text {intf }}\left(\left\{\phi_{\alpha}\right\}\right)+f^{\text {chem }}\left(\left\{\phi_{\alpha}\right\},\left\{\vec{c}_{\alpha}\right\}\right)\right\} d \Omega \\
f^{\text {inf }}\left(\left\{\phi_{\alpha}\right\}\right)=\sum_{\alpha=1}^{N} \sum_{\beta=\alpha+1}^{N}\left\{\frac{4 \sigma_{\alpha \beta}}{\eta}\left[-\frac{\eta^{2}}{\pi^{2}} \nabla \phi_{\alpha} \cdot \nabla \phi_{\beta}+\phi_{\alpha} \phi_{\beta}\right]\right\} \\
f^{\text {chem }}\left(\left\{\phi_{\alpha}\right\},\left\{\vec{c}_{\alpha}\right\}\right)= \\
\sum_{\alpha=1}^{N} \phi_{\alpha} f_{\alpha}\left(\vec{c}_{\alpha}\right)+\lambda\left[c-\sum_{\alpha=1}^{N}\left(\phi_{\alpha} c_{\alpha}\right)\right] \\
\sum_{\alpha=1}^{N} \phi_{\alpha}=1
\end{gathered}
$$

where $\phi_{\alpha}$ and $\phi_{\beta}$ are field variables of phases $\alpha$ and $\beta$, which are required to satisfy the equation. They further satisfy the constraints of equation (4). $\eta$ is the interface thickness, $\sigma_{\alpha \beta}$ is the interface energy between $\alpha$ and $\beta, f_{\alpha}\left(\vec{c}_{\alpha}\right)$ is the volume free energy density of phase $\alpha, \lambda$ is the Lagrange multiplier, $c_{\alpha}$ is the concentration of carbon in phase $\alpha$, and $N$ is the number of phases.

The phase field calculation function is shown in the equation. Where the variables $I_{\alpha}$, and $\Delta \tilde{g}_{\alpha \beta}$ are calculated from equations (6) and (7), respectively.

$$
\begin{gathered}
\dot{\phi}_{\alpha}=\frac{1}{N} \sum_{\beta=1}^{N} \dot{\psi}_{\alpha \beta}=\frac{1}{N} \sum_{\beta=1}^{N} \hat{\mu}_{\alpha \beta}\left(4 \eta / \pi^{2}\right) \cdot\left\{\left[\sigma_{\alpha \beta}\left(I_{\alpha}-I_{\beta}\right)+\sum_{\gamma=1, \gamma \neq \alpha, \gamma \neq \beta}^{N}\left(\sigma_{\beta \gamma}-\sigma_{\alpha \gamma}\right) I_{\gamma}\right]+\frac{\pi^{2}}{4 \eta} \Delta \tilde{g}_{\alpha \beta}\right\} \\
I_{\alpha}=\left\{\nabla^{2} \phi_{\alpha}+\frac{\pi^{2}}{\eta^{2}} \phi_{\alpha}\right\} \\
\Delta \tilde{g}_{\alpha \beta}=\Delta S_{\alpha \beta}\left[T^{r}+m_{\alpha \beta}\left(c_{\alpha}-c_{\alpha}^{r}\right)-T\right]
\end{gathered}
$$

where $\dot{\psi}_{\alpha \beta}$ is the interface field between phase $\alpha$ and $\beta, \Delta S_{\alpha \beta}$ is the transformation entropy, $T^{r}$ is the reference temperature, $m_{\alpha \beta}$ is slope of a corresponding line in phase diagram, $c_{\alpha}^{r}$ is the carbon concentration at the reference temperature $T^{r}$, and $T$ is transformation temperature.

Equation (8) is the diffusion equation, and the concentration field can be solved by combining equation (9) and equation (10).

$$
\begin{gathered}
\dot{c}=\nabla\left(\sum_{\alpha=1}^{N} M \nabla \frac{\delta F}{\delta c}\right)=\nabla \cdot \sum_{\alpha=1}^{N} \phi_{\alpha} D_{\alpha} \nabla c_{\alpha} \\
c=\sum_{\alpha=1}^{N} \phi_{\alpha} c_{\alpha} \\
c_{\alpha}=k_{\alpha \beta} c_{\beta}
\end{gathered}
$$

where $k_{\alpha \beta}$ represents the ratio of the concentration between $\alpha$ and $\beta . M$ is the chemical mobility and $D_{\alpha}$ is the diffusion coefficient of carbon in phase $\alpha$.

\subsection{Anisotropy and orientation models}

The anisotropic and orientation relationship models are introduced on the basis of the early nucleation model. Combining the growth patterns of ferrite and cementite, two different anisotropy models are used, respectively. The anisotropy model of ferrite is a common 4-symmetric model, as shown in Equation (11). The cementite is characterized by faceted anisotropy, its model is shown by equations (12) and (13), which was constructed by Steinbach ${ }^{[13]}$. The relevant parameters can find from references $[13,14]$.

$$
\begin{gathered}
\sigma(\theta)=1+C_{\text {Anis }} \cos 4 \theta \\
M(\theta)=M\left(k_{\text {kin }}+\frac{1-k_{\text {kin }}}{\kappa} \tanh \left(\frac{\kappa}{\tan \theta}\right) \tan \theta\right) \\
\sigma=\sigma_{0} k_{\mathrm{st}}^{2}\left(k_{\mathrm{st}}^{2} \cos ^{2} \theta+\sin ^{2} \theta\right)^{-1.5}
\end{gathered}
$$

where $C_{\text {Anis }}$ is anisotropy coefficient, $k_{\text {kin }}, \kappa$ and $k_{\text {st }}$ are constants, $\sigma_{0}$ is the initial interface energy.

In the process of austenite transformation into pearlite, two kids of orientation relationships are involved: one for the 
orientation relationship between austenite and ferrite/cementite, and the other for the orientation relationship between ferrite and cementite.

The relationship between austenite and ferrite is generally divided into: K-S relationship and Non K-S relationship. The $\mathrm{K}-\mathrm{S}$ relationship is more refined by Goro Miyamoto ${ }^{[3]}$ into: K-S relationship and Near K-S relationship. The definition of the interval is shown Table 1, where the K-S relationship is $\left([\overline{1} 10]_{\gamma} / /[1 \overline{1} 1]_{\alpha},(111)_{\gamma} / /(110)_{\alpha}\right)$.

Table 1: Definition of orientation between austenite and ferrite

$\begin{array}{cccc}\text { Orientation relationship } & \text { K-S } & \text { Near K-S } & \text { Non K-S } \\ \text { Orientation difference } & \Delta \theta \leqslant 5^{\circ} & 5^{\circ} \leqslant \Delta \theta \leqslant 15^{\circ} & >15^{\circ}\end{array}$

For the K-S orientation relationship, the interface energy can be processed by the Read Shockley equation ${ }^{[15,16]}$.

$$
\begin{gathered}
\sigma_{i i}=\left\{\begin{array}{lc}
\sigma_{i i}^{0} \frac{\Delta \theta}{\Delta \theta_{0}}\left(1-\ln \frac{\Delta \theta}{\Delta \theta_{0}}\right) & \Delta \theta<\Delta \theta_{0} \\
\sigma_{i i}^{0} & \Delta \theta \geq \Delta \theta_{0}
\end{array}\right. \\
\sigma_{\alpha \gamma}=\left\{\begin{array}{lr}
\frac{\sigma_{\alpha \gamma}^{0}}{2}+\frac{\sigma_{\alpha \gamma}^{0}}{2} \frac{\Delta \theta}{\Delta \theta_{0}}\left(1-\ln \frac{\Delta \theta}{\Delta \theta_{0}}\right) & \Delta \theta<\Delta \theta_{0} \\
\sigma_{\alpha \gamma}^{0} & \Delta \theta \geq \Delta \theta_{0}
\end{array}\right.
\end{gathered}
$$

where $i$ is one of ferrite, cementite or austenite phase, $\sigma_{i i}$ is interfacial energy of same phase between different grains. $\sigma_{\alpha \gamma}$ is interfacial energy between ferrite and cementite.

There are three main relationships between ferrite and cementite, namely Bagaryatsky, Isaichev, and Pitsch-Petch ${ }^{[17]}$, as shown in Table 2.

Table 2: Orientation relationship between ferrite and cementite

\begin{tabular}{lcc} 
Bagaryatsky & Isaichev & Pitsch-Petch \\
\hline$[100]_{c} / /[1 \overline{1} 0]_{\mathrm{f}}$ & {$[010]_{c} / /[111]_{\mathrm{f}}$} & {$[100]_{c} 2.6^{\circ}$ from $[\overline{3} 1 \overline{1}]_{\mathrm{f}}$} \\
{$[010]_{c} / /[111]_{\mathrm{f}}$} & $(101)_{c} / /(112)_{\mathrm{f}}$ & {$[010]_{c} 2.6^{\circ}$ from $[131]_{\mathrm{f}}$} \\
$(001)_{c} / /(112)_{\mathrm{f}}$ & & $(001)_{c} / /(21 \overline{5})_{\mathrm{f}}$
\end{tabular}

Note: Subscript $c$ and $f$ represent cementite and ferrite, respectively.
As the present work focused on the growth behavior of pearlite, it was assumed that ferrite and cementite have no orientation relationship, and were therefore not specifically considered.

\section{Results and discussion}

The initial phase field distribution is shown in Fig. 1, and the upper and lower parts are two different austenite grains. The middle region is the interface of two austenite grains $\gamma_{1}$ and $\gamma_{2}$. The initial position of pearlite nucleus is set at the center of the whole region. The critical nucleation concentrations of the ferrite and cementite are $3.2 \mathrm{~mol} \%$ and $3.72 \mathrm{~mol}^{\%}{ }^{[1]}$, respectively.

In the initial conditions, $200 \times 200$ mesh was used for the simulation. The grid size is $4 \mathrm{~nm}$ and the isothermal temperature is $970 \mathrm{~K}$. The simulation parameters are shown in Table 3. The boundary conditions are set to be adiabatic.

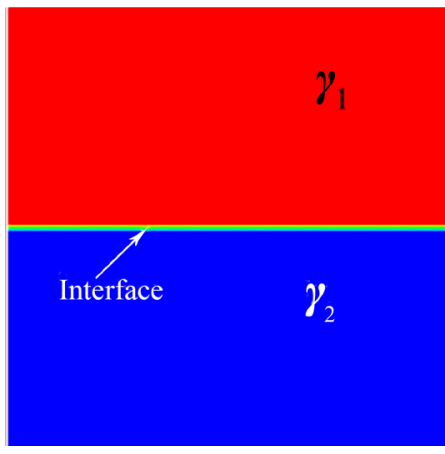

Fig. 1: Initial conditions

\subsection{Effect of anisotropy on pearlite}

The anisotropy of ferrite and cementite is represented by a 4-symmetric model. The orientations of two adjacent austenite grains are set as $0^{\circ}$ and $45^{\circ}$, respectively. The nucleus orientation of pearlite varies from $0^{\circ}$ to $22.5^{\circ}$.

Due to the anisotropy of ferrite and cementite, the growth morphologies of ferrite and cementite are different. Figure 2 shows the growth state of the pearlite from the boundary into the austenite grains under anisotropic conditions at $0.10 \mathrm{~s}$, where the orientation of austenite $\gamma_{1}$ is $0^{\circ}$, and the orientation of austenite $\gamma_{2}$ is $45^{\circ}$. It can be clearly found that the growth direction of the

Table 3: Simulation parameters ${ }^{[18]}$

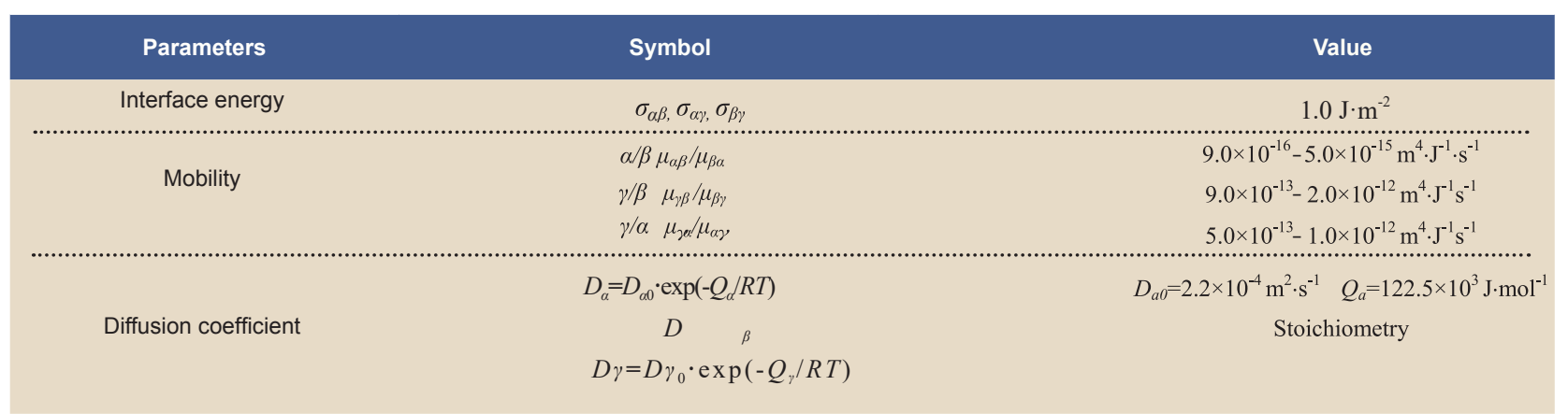

Note: $\alpha$ denotes ferrite, $\beta$ denotes cementite, and $\gamma$ denotes austenite. 
pearlite into the austenite grains on both sides of the boundary is different due to the different orientations of the austenite grains on both sides. Figure 2(a) shows that the growth direction of the pearlite in $\gamma_{1}$ is perpendicular to the grain boundary, that is, the angle between the growth direction of pearlite and the vertical direction is $0^{\circ}$, which is equal to the orientation difference between the austenite $\gamma_{1}$ and the pearlite. However, the angle between the growth direction of pearlite in $\gamma_{2}$ and the grain boundary of austenite is $45^{\circ}$, which is equal to the orientation difference between the austenite $\gamma_{2}$ and the pearlite. Figures 2(b), (c), (d), (e) and (f) have the same rules, as shown in Table
4. However, the growth direction of pearlite will deviate with the growth of pearlite, as the second angle marked in Fig. 2(e) and (f). When the cementite lamellae at the left side began to grow, the growth direction of cementite lamellae at right side deviated from the orientation angle. This results in a large spacing between the two cementite lamellae. In order to reduce the lamellar spacing, the growth direction of cementite lamellae at the right side tends to shift toward the center of the two cementite lamellae. This phenomenon indicates that the growth direction of the pearlite lamellae is related to the orientation of the pearlite lamellae and the lamellar spacing of pearlite.

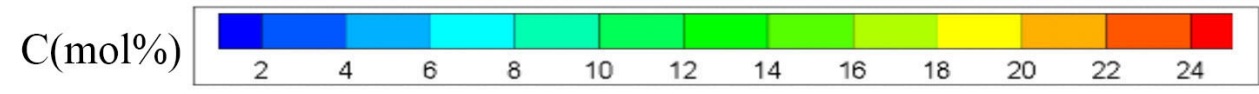

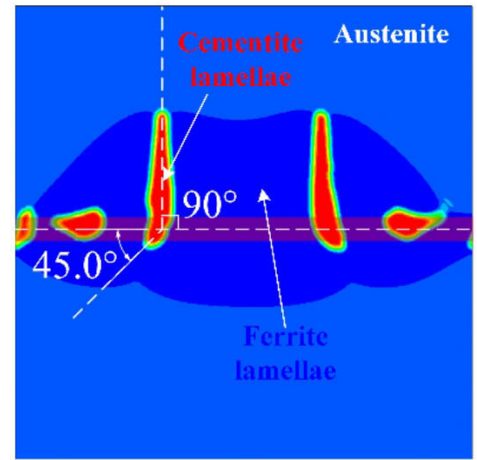

(a) Orientation is $0^{\circ}$

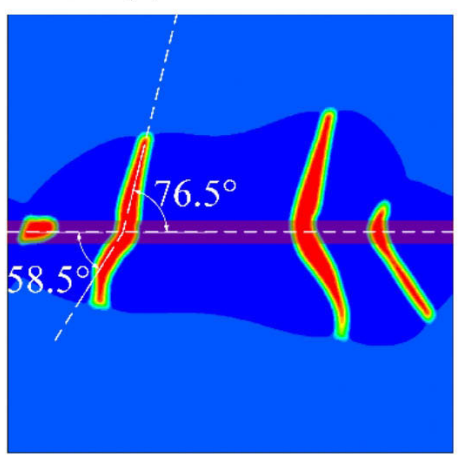

(d) Orientation is $13.5^{\circ}$

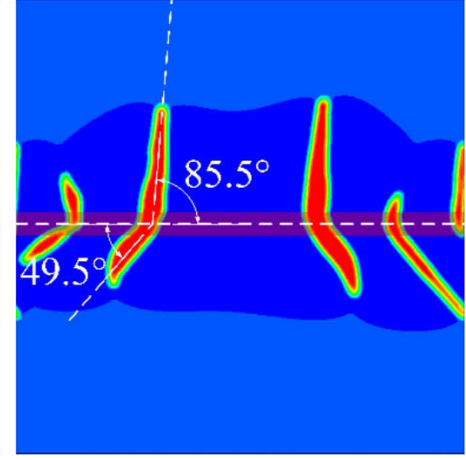

(b) Orientation is $4.5^{\circ}$

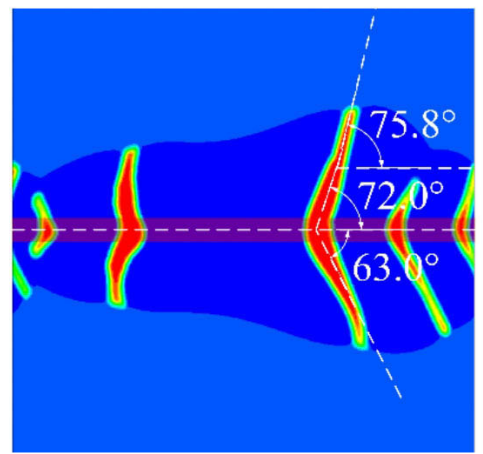

(e) Orientation is $18.0^{\circ}$

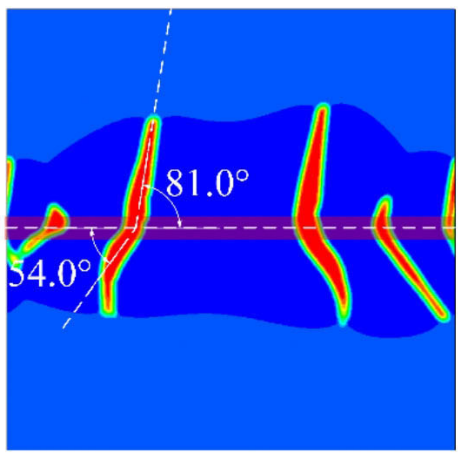

(c) Orientation is $9.0^{\circ}$

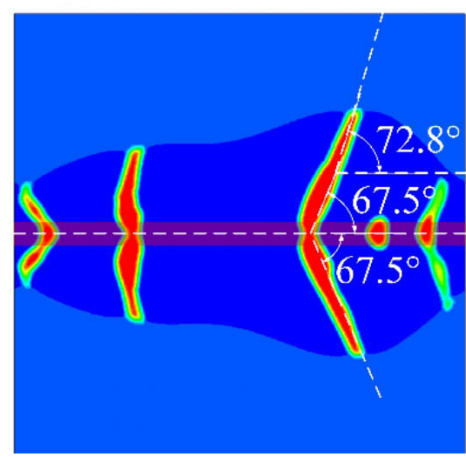

(f) Orientation is $22.5^{\circ}$

Fig. 2: Concentration distribution of pearlite with different orientations at $0.10 \mathrm{~s}$

Table 4: Angle between growth direction and horizontal direction in austenite grains

\begin{tabular}{|c|c|c|c|c|c|c|}
\hline Pearlite orientation & $0^{\circ}$ & $4.5^{\circ}$ & $9.0^{\circ}$ & $13.5^{\circ}$ & $18.0^{\circ}$ & $22.5^{\circ}$ \\
\hline$\gamma_{1}\left(0^{\circ}\right)$ & $90.0^{\circ}$ & $85.5^{\circ}$ & $81.0^{\circ}$ & $76.5^{\circ}$ & $72.0^{\circ}$ & $67.5^{\circ}$ \\
\hline$\gamma_{2}\left(45^{\circ}\right)$ & $45.0^{\circ}$ & $49.5^{\circ}$ & $54.0^{\circ}$ & $58.5^{\circ}$ & $63.0^{\circ}$ & $67.5^{\circ}$ \\
\hline
\end{tabular}

\subsection{Effect of orientation relation on pearlite}

According to the experiments of Miyamoto ${ }^{[3]}$, the orientation difference between the pearlite lamellae and the austenite is mainly concentrated between $0^{\circ}-25^{\circ}$. Considering that the orientation of two adjacent austenite grains is $0^{\circ}$ and $25^{\circ}$, the nucleus orientation of the pearlite lamellae is set to $5^{\circ}, 10^{\circ}$, $15^{\circ}$ and $20^{\circ}$, respectively. Due to the different orientations of pearlite, the pearlite grows into different morphologies.

Figure 3 shows the growth morphology of pearlite in different orientations. The grid region is the boundary between two austenite grains. The interface energy between ferrite and austenite would vary drastically with the orientation when the 
orientation relationship is considered. When the orientation difference is less than $5^{\circ}$, the lamellar ferrite does not grow directly into the austenite grains, but grows in the form of Widmanstatten pattern ferrite or boundary ferrite. When the orientation difference is greater than $5^{\circ}$, it grows into the austenite grains. The pearlite in the two austenite grains in Fig. 3 (a) and (b) shows two different growth morphologies. At the same time, the cementite in Fig. 3(a) and (d) is more likely to grow along the austenite boundary. In the vicinity of the austenite boundary, there are ferrite and cementite lamellae parallel to the austenite boundary. The ferrite may be firstly precipitated, then cementite would be precipitated from the boundary between ferrite and austenite. The orientation difference between the austenite and pearlite is $10^{\circ}$ and $15^{\circ}$ for Fig. 3(b) and (c), so there was very little difference between the growth rate of pearlite on both sides of the austenite boundary.

In particular, it is noted that the austenite grain boundary varies with the different orientations of pearlite. At the beginning, the austenite grain boundary is a horizontal straight line. With the pearlite growing in the austenite grain, the grain junction is changed from a two-phase junction to a three-phase junction. The boundary evolution mechanism in Fig. 4 shows that only $\sigma_{\gamma_{1} \gamma_{2}}$ exists at initial state and the interface of two austenite grains will not bend. With the formation of the ferrite nucleus, the interface [ $\sigma_{\gamma_{1} \alpha}$ and $\sigma_{\gamma_{2} \alpha}$, as shown in Fig. 4(b) and (e)] between austenite and ferrite appears. Since the two austenite grains are in different orientations, $\sigma_{\gamma_{1} \alpha}$ and $\sigma_{\gamma_{2} \alpha}$ have different values, and they vary with the normal values of the interface at the junction point. At this time, if $\sigma_{\gamma_{1} \gamma_{2}}$ still remains horizontal, the three interface energies would be unbalanced at the junction. In view of $\sigma_{\gamma_{1} \alpha}<\sigma_{\gamma_{2} \alpha}$, and the direction of interface energy between austenite $\gamma_{1}$ and ferrite being approximately horizontal, the vector sum of $\sigma_{\gamma_{1} \alpha}$ and $\sigma_{\gamma_{2} \alpha}$ is as shown in Fig. 4(c) and (f). In order to ensure balance, the direction of $\sigma_{\gamma_{1} \gamma_{2}}$ is the reverse of the vector sum. As the growth continues, the interface will move toward the inside of

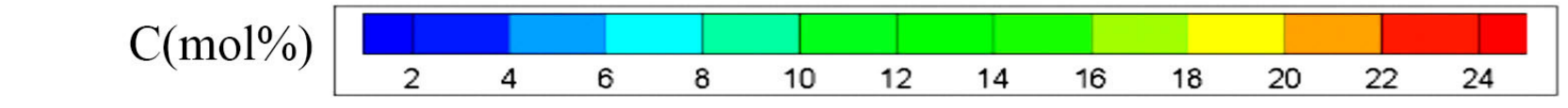

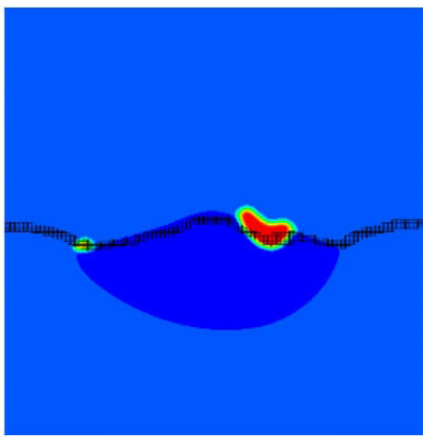

(a) Orientation is $5^{\circ}$

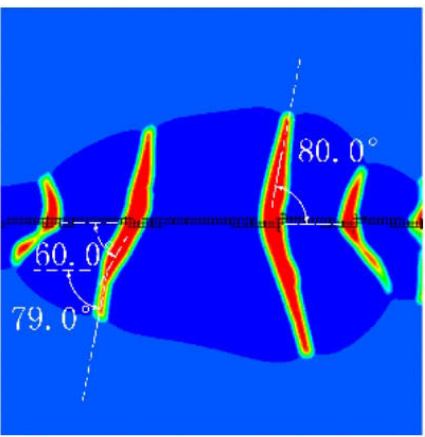

(b) Orientation is $10^{\circ}$

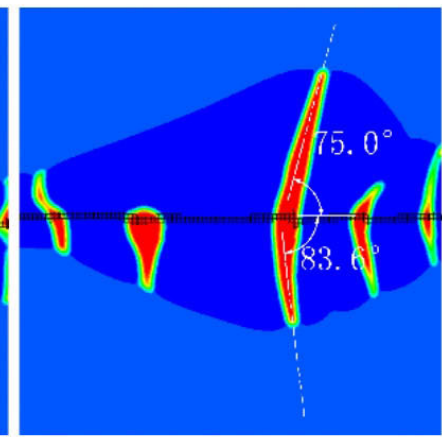

(c) Orientation is $15^{\circ}$

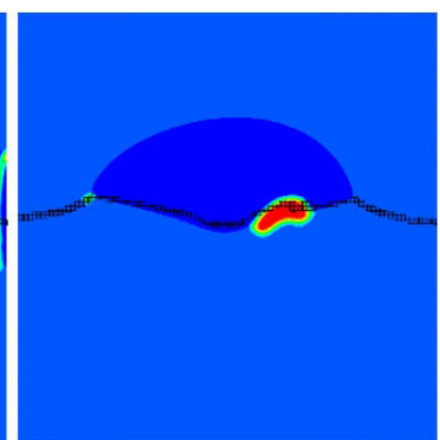

(d) Orientation is $20^{\circ}$

Fig. 3: Growth morphology of pearlite in different orientations when K-S relationship is introduced at $0.116 \mathrm{~s}$

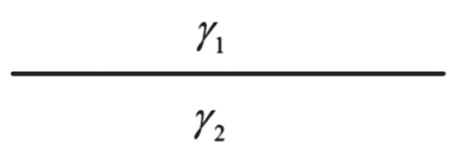

(a)

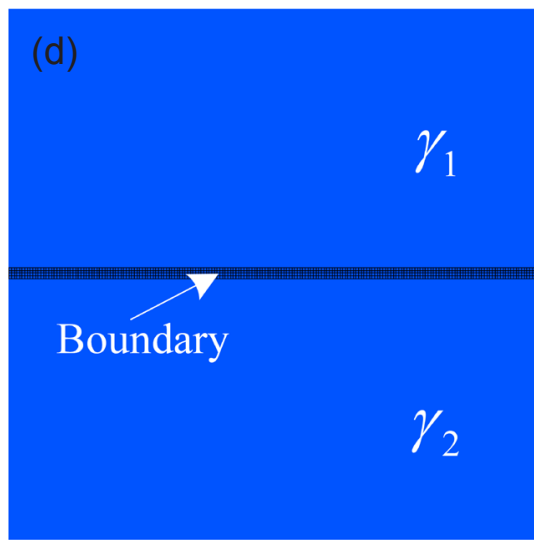

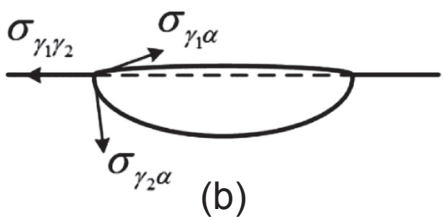

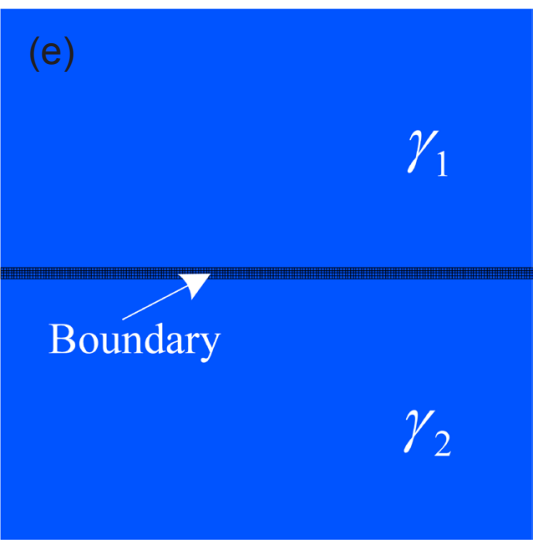

Fig. 4: Boundary evolution mechanism
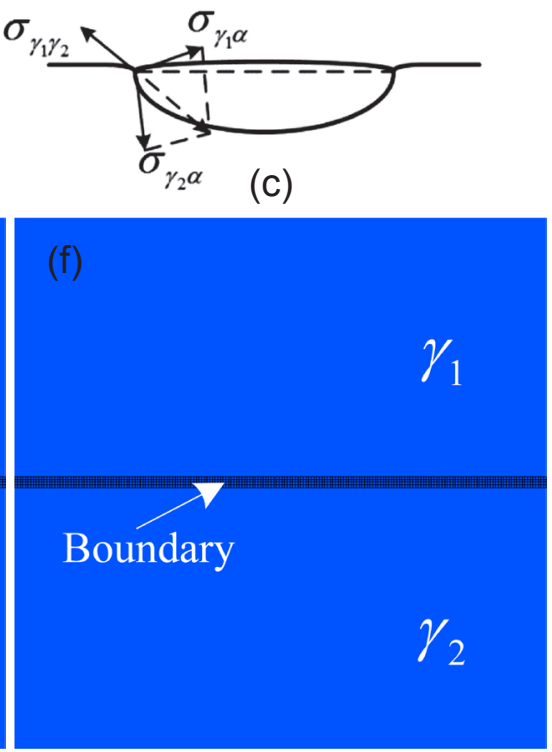
the grain, eventually showing such morphology as shown in Fig. 3(a) and (d).

\subsection{Effect of nucleation positions on pearlite}

The nucleation site of the above pearlite growth process is at the austenite grain boundaries, but the pro-eutectoid phase will appear in the process of the actual growth of pearlite, and preferentially nucleates and grows at the austenite grain boundary. Therefore, the subsequent nucleation sites are mostly at the interface of the pro-eutectoid phase and the austenite phase. However, for the alloys of the different compositions, the distribution of impurity particles inside the austenite grains would be very different. The nucleation induced by the inclusion in grains or boundaries is not considered herein.

When the nucleus location is set at the interface of the austenite phase and the pro-eutectoid phase or austenite phase, the K-S orientation relationship is not changed, as shown in Fig. 5. Figure 5 (a) and (d) show unilateral austenite growth, whereas (b) and (c) show lamellar growth. Compared with the growth morphology of pearlite with different orientations in Fig. 3 , it can be found that the pearlite at the nucleus location that set at the interface of the austenite phase and the pro-eutectoid phase or austenite phase can develop the bifurcation mechanism (that is, a ferrite lamella is divided into two ferrite lamellae) of lamellar growth, as shown in the virtual box of Fig. 5(b). The growth rate of the lamellar region would decrease when the lamellar spacing is large. The carbon atoms precipitated from lamellar ferrite will be hoarded here until they are higher than the critical nucleation concentration, then forming the nucleus of cementite. Morphological characteristics are manifested as bifurcation phenomena.

In view of the actual situation, there will be granular pearlite structure, but it cannot form a granular pearlite structure when the nucleation site is at the interface of two austenite grains. When the nucleus location is set at the interface of the austenite phase and the pro-eutectoid phase or austenite phase, granular pearlite can form in the simulation results, that is, a plurality of granular cementite distribute in the ferrite matrix.

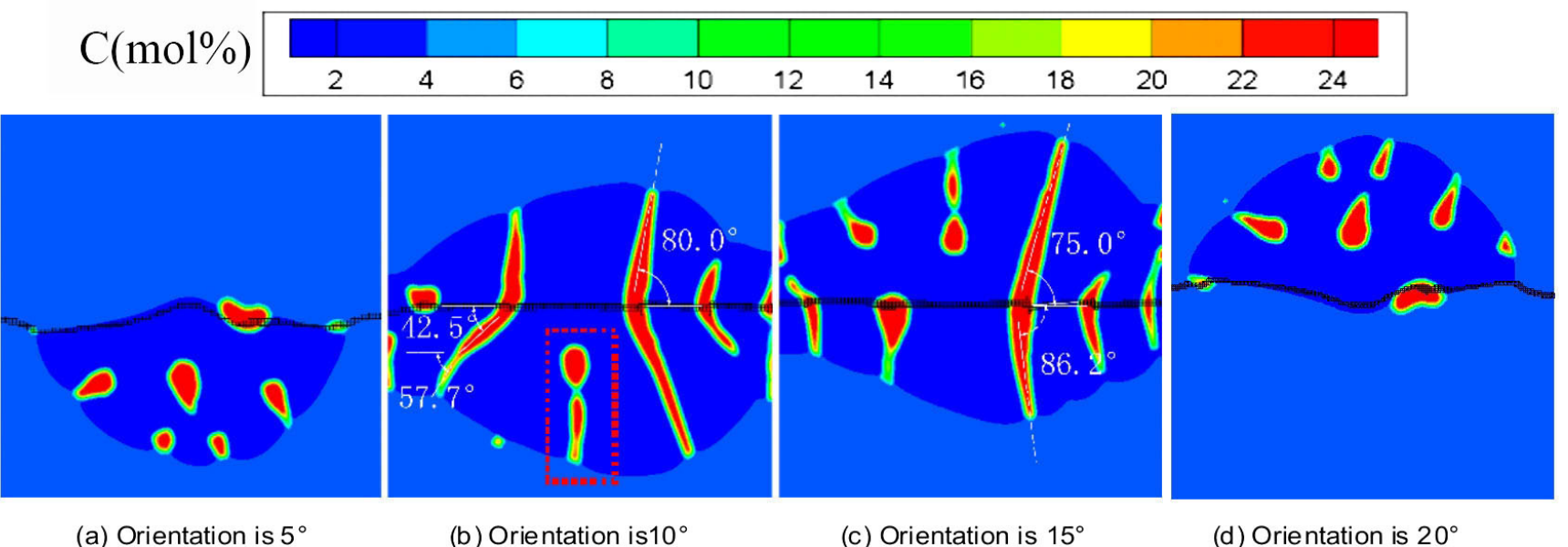

Fig. 5: Growth morphologies of pearlite at different orientations at $0.128 \mathrm{~s}$

\subsection{Experimental verification}

It is difficult to obtain the mechanism of microstructure evolution during the eutectoid phase transformation by experiment. In the previous study on the formation of grain boundary ferrite in eutectoid and hypereutectoid pearlitic steels by Miyamoto et al ${ }^{[3]}$, there are four microstructures, namely: boundary ferrite, Widmanstatten ferrite, granular pearlite, and lamellar pearlite when the carbon content is $0.75 \mathrm{wt} . \%$ and the transition temperature is $873 \mathrm{~K}$, as shown as in Fig. 6. It was found that the four microstructures are determined by the orientation relationship between the austenite and pearlite. That is, when the orientation difference is less than or equal to $5^{\circ}$, Widmanstatten ferrite and boundary ferrite are liable to appear. When the orientation difference is more than $5^{\circ}$ and less than or equal to $15^{\circ}$, discrete pearlite is liable to produce. When the orientation difference is more than $15^{\circ}$, lamellar pearlite is liable to produce.
In the study, to verify the accuracy of the model indirectly, the simulation initial conditions were set according to the actual composition and temperature of the above experiment, with only $0.8 \mu \mathrm{m} \times 0.8 \mu \mathrm{m}$ region being simulated due to the simulation area limitation. Figure 7 shows the simulation results with the same conditions as Fig. 6. The orientation of lamellar pearlite is $4.5^{\circ}$, the orientation difference between austenite $\gamma_{1}$ and the lamellar pearlite is $4.5^{\circ}$, the orientation difference between austenite $\gamma_{2}$ and the lamellar pearlite is $15.5^{\circ}$.

Figures 6 and 7 show two kinds of microstructure with different morphologies. The left side of Fig. 6 (a) is the pearlite, the right side is the Widmanstatten ferrite and pearlite. The left side of Fig. 6 (b) is the lamellar pearlite, the middle boundary is boundary ferrite, and the right side is the martensite which corresponds to the austenite of original microstructure. The lower part of Fig. 7 is lamellar pearlite, the middle boundary is the boundary ferrite, and the upper part is the austenite that 

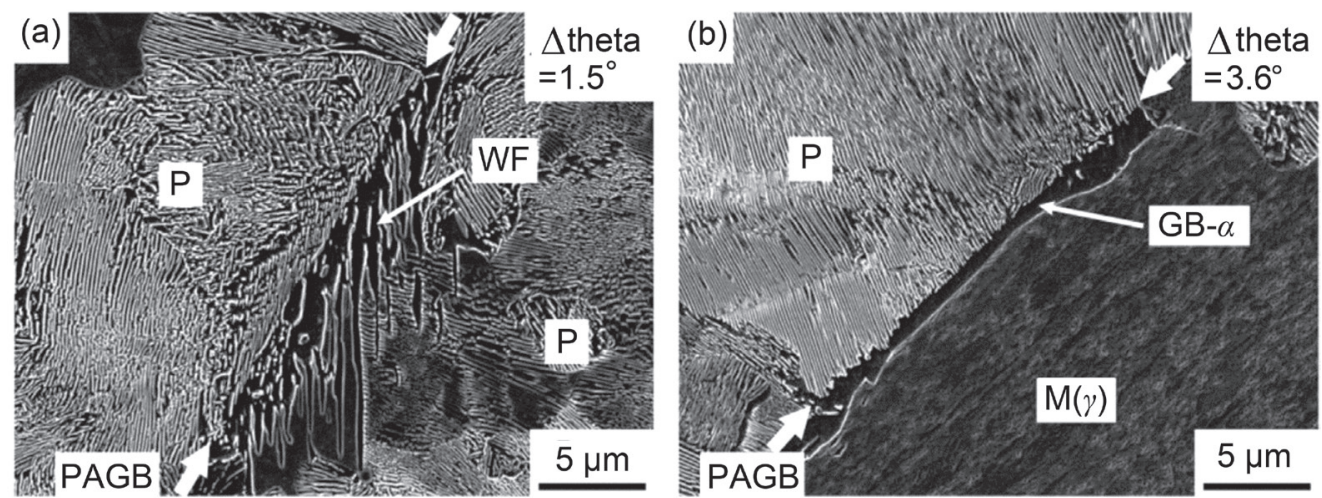

Fig. 6: Metallographic microstructure formed in $0.75 \mathrm{wt} . \%$ alloy transformed at $873 \mathrm{~K}$ for $7 \mathrm{~s}^{[3]}$

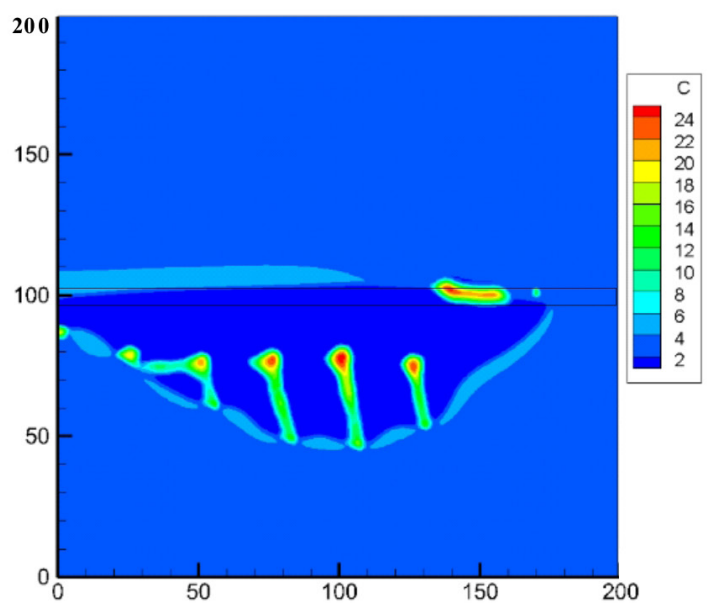

(a) Concentration distribution

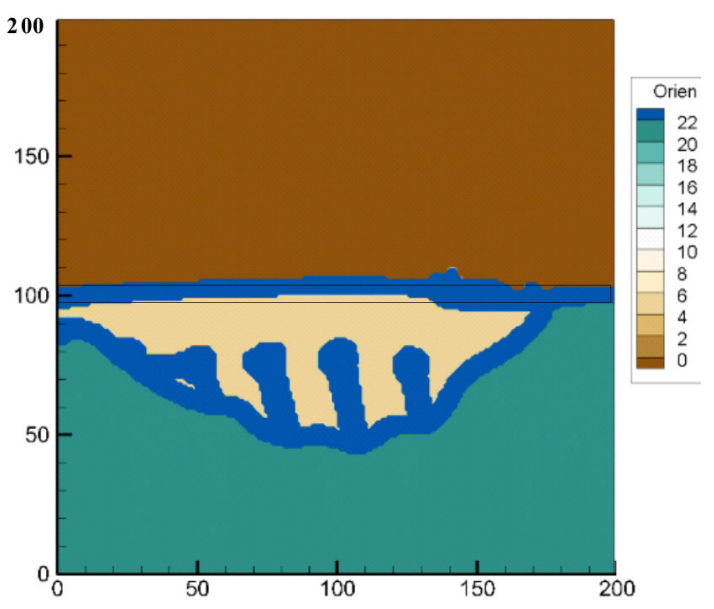

(b) Orientation distribution

Fig. 7: Simulation results at $873 \mathrm{~K}$ for $7 \mathrm{~s}$

has not transformed. The reason for the formation of boundary ferrite and Widmanstatten ferrite is that the orientation difference between the austenite and the ferrite is less than $5^{\circ}$ [1.5 ${ }^{\circ}$ for Fig. 6 (a), $3.6^{\circ}$ for Fig. 6 (b), and $4.5^{\circ}$ for Fig. 7]. It can be found that the simulation results are in line with the experimental conclusion. Boundary ferrite is easy to produce when the orientation difference is less than $5^{\circ}$ (austenite $\gamma_{1}$ ), here, as the characteristics of Widmanstatten ferrite were not set, then Widmanstatten ferrite will not appear. Lamellar pearlite is easy to produce when the orientation difference is greater than $15^{\circ}$ (austenite $\left.\gamma_{2}\right)$

\section{Conclusions}

By introducing anisotropy and orientation relationships, the growth of the pearlite lamellae was studied by multi-phase field method. It was found that the simulation results were in agreement with the experimental results. Therefore the following conclusions can be drawn:

(1) The growth direction of the pearlite lamellae is related to the orientation and spacing of the pearlite lamellae. The growth direction of the pearlite lamellae is deflected along with the adjustment of lamellar spacing, and the deflection angle is equal to the orientation difference between the pearlite lamellae and austenite.

(2) When the orientation difference between the pearlite lamellae and austenite is less than $5^{\circ}$, the pearlite lamellae does not grow into the austenite grains, but grows in the form of boundary ferrite. When the orientation difference between the pearlite lamellae and austenite is greater than $5^{\circ}$, the pearlite lamellae grows into the austenite grains.

(3) When the nucleus of ferrite lamellae or cementite lamellae is located at the interface of austenite and ferrite, cementite or austenite, the K-S orientation relationship is not changed. At the same time, the nucleus at the interface of austenite and ferrite, cementite or austenite can induce the bifurcation of thick ferrite.

\section{References}

[1] Zhang D, Yin Y, Zhou J, et al. Phase field study of microstructure evolution in eutectoid phase transformation - I nucleation. Archives of Foundry Engineering, 2017, 17(3): 155-162.

[2] Zhi X, Liu J, Xing J, et al. Effect of cerium modification on 
microstructure and properties of hypereutectic high chromium cast iron. Materials Science and Engineering: A, 2014, 603: 98103.

[3] Miyamoto G, Karube $Y$, Furuhara T. Formation of grain boundary ferrite in eutectoid and hypereutectoid pearlitic steels. Acta Materialia, 2016, 103: 370-381.

[4] Smith C S. Microstructure. Trans. ASM, 1953, 45: 533.

[5] Guo Z, Kimura N, Tagashira S, et al. Kinetics and Crystallography of intragranular pearlite transformation nucleated at (MnS plus VC) complex precipitates in hypereutectoid FeMn-C alloys. ISIJ International, 2002, 42(9): 1033-1041.

[6] Furuhara T, Yamaguchi J, Sugita N, et al. Nucleation of Proeutectoid Ferrite on Complex Precipitates in Austenite. ISIJ International, 2003, 43(10): 1630-1639.

[7] Furuhara T, Shinyoshi T, Miyamoto G, et al. Multiphase crystallography in the nucleation of intragranular ferrite on MnS plus $\mathrm{V}(\mathrm{C}, \mathrm{N})$ complex precipitate in austenite. ISIJ International, 2003, 43(12): 2028-2037.

[8] Wiessner M, Angerer P, Prevedel P, et al. Advanced X-ray Diffraction Techniques for Quantitative Phase Content and Lattice Defect Characterization during Heat Treatment of High Speed Steels. BHM Berg- und Hüttenmännische Monatshefte, 2014, 159(9): 390-393.

[9] Cheng L, Wu K M. New insights into intragranular ferrite in a low-carbon low-alloy steel. Acta Materialia, 2009, 57(13): 37543762.

[10] Cheng L, Wan X L, Wu K M. Three-dimensional analysis of ferrite allotrimorphs nucleated on grain boundary faces, edges and corners. Materials Characterization, 2010, 61(5): 580-583.

[11] Cheng $L$. The nucleation, three dimensional morphology and growth kinetics of ferrite in low carbon high strength microalloyed steels. Wuhan: Wuhan University of Science and Technology, 2013. (In Chinese)

[12] Wang Z C, Li W, Guo Z H, et al. Simulation of Ferrite/Cementite Interface with Bagaryatsky Orientation Relationship. Journal of Shanghai Jiao Tong University, 2005, 39(1): 14-17. (In Chinese)

[13] Steinbach I, Pezzolla F, Prieler R. Grain selection in faceted crystal growth using the phase field theory. Minerals, Metals and Materials Society, Warrendale, PA (United States), 1995.

[14] Eggleston J J, Mcfadden G B, Voorhees P W. A phase-field model for highly anisotropic interfacial energy. Physica $D$ Nonlinear Phenomena, 2001, 150(1): 91-103.

[15] Yamanaka A, Takaki T, Tomita Y. Multi-phase-field modeling of diffusive solid phase transition in carbon steel during continuous cooling transformation. Journal of Crystal Growth, 2008, 310(79): $1337-1342$.

[16] Read W T, Shockley W. Dislocation Models of Crystal Grain Boundaries. Physical Review, 1950, 78(3): 275-289.

[17] Mangan M A, Shiflet G J. The Pitsch-Petch orientation relationship in ferrous pearlite at small undercooling. Metallurgical and Materials Transactions A, 1999, 30(11): 27672781.

[18] Nakajima K, Apel M, Steinbach I. The role of carbon diffusion in ferrite on the kinetics of cooperative growth of pearlite: A multiphase field study. Acta Mater., 2006, 54(14): 3665-3672. 